\title{
EL IMPACTO AMBIENTAL DEL CAFÉ EN LA HISTORIA COSTARRICENSE
}

Enero de 1994

\author{
Carlos Granados \\ Departamento de Geografía \\ Universidad de Costa Rica
}

El igual que diversos países de Centroamérica, el Caribe y el resto de América Latina, Costa Rica cuenta con una larga trayectoria cafetalera. Como en Brasil, Colombia, Guatemala o El Salvador, la caficultura en Costa Rica a constituido un factor primario de generación de empleo, comercio exterior y divisas. Pero, ha diferencia de otros países donde el grano se ha cultivado, en Costa Rica el café (conjuntamente con el banano) ha constituido la columna vertebral de la historia económica nacional. El café, cuya producción antecede en más de medio siglo a la del banano, permitió al naciente estado de Costa Rica superar la situación de anarquía que trajo el fin de la colonia. Su cultivo posibilitó el establecimiento de una economía nacional, representó una salida ante la fragmentación económica regional que la corona había heredado, e incorporó a Costa Rica al mercado mundial. Mas que un mero artículo de consumo y exportación, el café se constituyó en un forjador de la economía, la cultura y la nacionalidad costarricense.

Precisamente por ese papel estructurador de la actividad cafetaleras, ha existido una visión sumamente positiva de lo que los costarricenses conocen cono el "grano de oro". Se tiende a pensar, no sin razón, que el café ha cumplido una especie de misión civilizadora y que la forja de la patria ha sido asunto, primero y ante todo, de los cafetaleros. Si Costa Rica ha sido vista en el exterior como una "Banana Republic", los costarricenses prefieren considerarse hijos de una patria cafetalera. Muchos de los problemas sociales (división en clases de la sociedad), económicos (monocultivismo) y políticos (surgimiento de una élite cafetalera dominante, dependencia externa), que el café creó o profundizó, son con frecuencia minimizados, para exaltar los atributos positivos de la sociedad cafetalera costarricense.

Aunque la literatura sobre el café en Costa Rica es abundante, fue necesario esperar hasta 1976 para recibir un tratamiento crítico y sistemático del papel del café en la historia del país. El Café y el Desarrollo Histórico-Geográfico del Café en Costa Rica, de Carolyn Hall, representó el primer intento por llenar un vacío que un literatura, generalmente justificativa, había dejado sobre el desarrollo del café (Hall:1991). Hoy, casi veinte años después, la obre de Hall continúa siendo de lectura obligatoria para aquellos interesados en la comprensión de las transformaciones económicas y territoriales inducidas por el café.

Como resultado de la actitud elogiosa con que la materia ha sido tratada, el tema del impacto ambiental de la caficultura tampoco ha recibido mayor interés. Incluso en la obra de Hall geógrafa de profesión, las referencias al café como problema ambientas, que las hay, son escasas. Esto último no es de extrañar si se considera que el movimiento mundial ecologista era apenas incipiente cuando el libro fue escrito y, lo que es mas importante, si se argumenta como se hará mas adelante, que el café en Costa Rica no 
constituía una amenaza ambiental severa en esos días. Así, aunque la producción cafetalera haya sido señala como responsable de numerosas complicaciones ambientales, la conciencia social de estos males, por los que el país paga hoy día un elevado precio, es todavía muy restringida. Salvo raras excepciones, la investigación se ha dirigido a mejorar la productividad cafetalera, dejando al margen la consideración de sus repercusiones ambientales. Por eso el conocimiento es escaso y fragmentario.

Hoy, a 150 años de establecida la agricultura en Costa Rica, el café vive uno de los peores trances de su historia, que bien podría degenerar en una crisis terminal . No obstante un constante incremento en la productividad, o quizás por eso mismo, los

precios y los ingresos del café han caído dramáticamente en la década de 1980. Habiendo constituido la columna vertebral del país por mas de un siglo, actualmente el café no representa ni un $2 \%$ del Producto Interno Bruto, genera poco empleo, pobres divisas y mas que limitados ingresos al estado. Paradójicamente, el impacto ambiental y los costos sociales derivados son crecientes, permitiendo incluso hablar de una crisis ambiental del orden cafetalero.

El presente ensayo tiene como propósito realizar una consideración general del impacto ecológico de la caficultora en los anales del país, particularmente durante los tres últimos decenios. La tesis de este ensayo es que, durante los últimos treinta años, período en que Costa Rica se incorpora a la llamada Revolución Verde, se produce una reestructuración de los agrosistemas cafetaleros, cuyas consecuencias en el ambiente adquieren proporciones alarmantes. A pesar de que la evidencia empírica precisa sobre el asunto sólo existe para años recientes, se intentará discutir las eventuales repercusiones ambientales en etapas anteriores, porque sólo así puede dibujarse el marco comparativo para evaluar la situación presente.

\section{EL CAFÉ Y EL MEDIO TROPICAL}

Al considerar el impacto ambiental del café, una pregunta se impone de inmediato: ¿cuáles eran las características de los ambientes que fueron asimilados por la actividad cafetalera? Debe en primer lugar verificarse el hecho de que los ambientes en que la caficultora se enraizó eran, salvo en los años introductorias de la caficultora, tierras 
"vírgenes", es decir, dominios que anteriormente no formaban parte del ecúmeno hispanoamericano, Las labores entonces, se desarrollan a expensas de medios poco alterados, cubiertos previamente por densos bosques tropicales. Así, evaluar las consecuencias ambientales del café presupone comprender el dinamismo del bosque tropical, dinamismo al que aquí sólo nos referimos brevemente.

Una de las grandes paradojas del medio tropical húmedo es aquella de que, siendo un medio tan frágil, es capaz de sustentar el exuberante bosque tropical s, de una diversidad florística y un volumen de biomasa sin parangón. En los trópicos, los materiales rocosos son rápidamente degradados por la acción del clima, por las pertinaces lluvias y por la actividad física y biológica que las elevadas temperaturas permiten y propician. La formación de suelos es tan intensa como constante. Los gruesos suelos tropicales se revisten de selvas protectoras, y son fácil presa de la erosión en ausencia de una adecuada capa vegetal. La asociación suelo-flora es particularmente crítica en el trópico húmedo. El suelo consiente el crecimiento de una macro-vegetación. El bosque, a su vez, protege el suelo de las lluvias torrenciales. El ciclo de la vida es constante en el bosque tropical; el letargo que caracteriza a la vegetación de las zonas templadas, durante los rigurosos meses invernales, es desconocido en el trópico. Aquí la vegetación germina y muere a cada instante. De la hojarasca de la selva brotan diariamente toneladas de humus, que son desintegradas e incorporadas a los suelos con igual celeridad. Los cambios se suceden velozmente, pero existe una especie de punto de equilibrio: el vulnerable suelo soporta una flora lujuriosa, de especies que se cuentan en miles por kilómetro cuadrado; el follaje de los árboles por su parte, atenúa el impacto de las lluvias y sus extensas raíces mantienen el suelo en su lugar. La diversidad de especies es adaptación a la multiplicidad de nutrientes; garantiza, a su vez, una permanente cobertura, porque cada especie tiene su propio calendario de crecimiento. Tal es, groso modo, el secreto del bosque tropical.

En su condición original, el café (Coffea arabica) fue una especie del bosque tropical. La planta es nativa de las selvas de Etiopía, en donde crecía como parte del estrato arbustivo de la floresta. (figura1). 


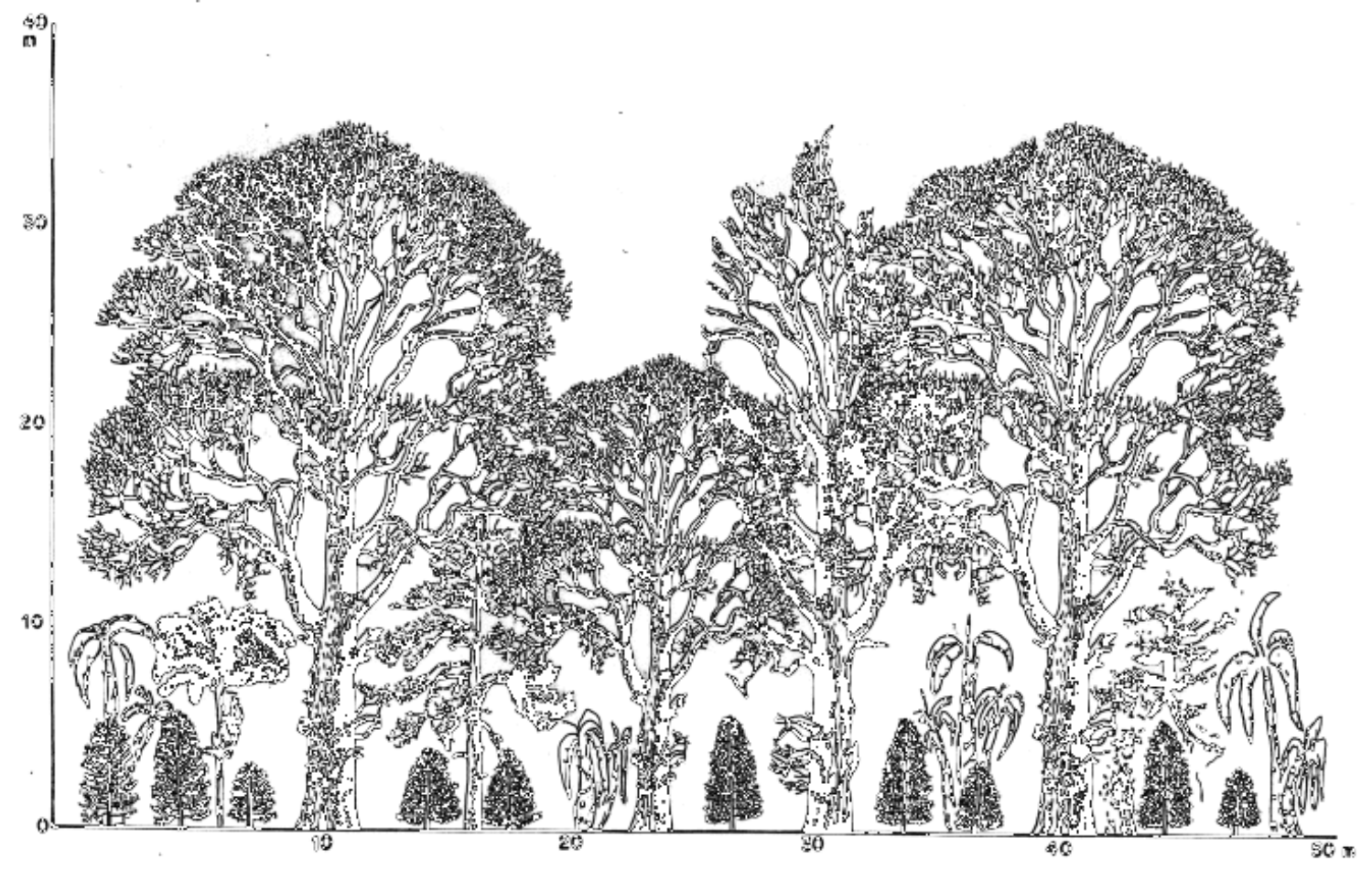

Fuente: Fournier:1980:16

Como ilustra la Figura 1, en el bosque tropical el café comparte el ambiente con una pluralidad de especies, siendo por ello corto el número de cafetos en el medio. El paso del ecosistema natural, del que el café alguna vez fue parte, al agro-sistema que llamamos cafetal, supone, necesariamente, mutaciones importantes. El aumento del número de plantas, en el estado bajo de un bosque, supone un clareo de la floresta, de modo que más radiación solar alance el suelo y permita el crecimiento del café. Crecerá más café, pero también lo hará el estrato herbáceo, que antes no podía prosperar por la falta de luz a ras del suelo. Las hierbas compiten con el café, y es necesario controlarlas con métodos artificiales. La supresión total o parcial del estrato arbóreo, a su vez, modifica el ciclo del agua, porque se pierde capacidad de almacenaje en el follaje y porque mas lluvia impacta el suelo, aumentándose la escorrentía superficial y los riesgos de erosión. El intercambio de materia entre el suelo y vegetación se altera con la especialización florística, etc.

La conversión de la selva en cafetal, de por sí, habla de un poderoso impacto en el ambiente, porque el monocultivo es la negación del trópico. El trópico propende 
naturalmente a la diversidad, y todo intento de especializar lo que por naturaleza está llamado a ser diverso implica un gran trastorno. Desde ese punto de vista, puede decirse que el café, a pesar idílica imagen suya que consta en la imaginación de los costarricenses, ha representado una amenaza ambiental. Esta constatación , sin embargo, nos dejaría en un nivel muy general de pensamiento, porque el desarrollo cafetalero costarricense ha conocido múltiples etapas, en cada una de las cuales observamos secuelas ambientales específicas.

\section{CAFÉ Y AMBIENTE EN LA HISTORA DE COSTA RICA}

El café fue traído a costa Rica a principios del siglo pasado, pero sólo conoció su auge hacia 1850. las tierras altas del Valle central, donde había estado centrada la población y la vida colonial costarricense, fueron la cuna de la caficultura. En estas tierras, a elevaciones promedio de unos 1200 metros, en los ricos suelos volcánicos del fondo del valle, bien drenados, de suaves pendientes convexas, y bañados por precipitaciones moderadas (entre 1500 y 2000 milímetros anuales) los primeros cafetales encontraron las condiciones óptimas para su desarrollo.

La vegetación natural de la zona era la típica del piso premontano húmedo, el bosque del mismo nombre ha sido detalladamente descrito por Holdridge (1971), se trataba de un bosque semicaducifolio, compuesto por varios estratos. El estrato superior en el que se presentaban las especies caducifolias, alcanzaba entre 20 y 25 metros, y presentaba copas mas bien chatadas y de gran extensión lateral, por lo que brindaba un alto porcentaje de cobertura. Un segundo estrato estaba constituido por árboles bajos perennifolios y arbustos, de una gran riqueza florística. Completaban el paisaje el estrato inferior de arbustos e hierbas. En esta arquitectura forestal descollaban árboles cono el ron ron (Astronium graveolens), el pochote (Bombacopsis quinata), el higuerón (Picus sp), el cenízaro (Anarcadium excelsum), el guanacaste (Enterolobuim cyclocarpum), la ceiba (Ceiba pentandra) y el laurel (Cordia alliodora). En conjunto, se trataba de un bosque denso y variado, que ofrecía una cobertura espesa y constante del suelo, que era profundo, oscuro y rico en materia orgánica (Holdridge: 1971: 334-342). 
Desde su inicio, y hasta 1850, la siembra del café estuvo circunscrita a los alrededores de San José, principal ciudad y capital de la República. Durante la segunda parte del siglo, el área de cafetales se expandió considerablemente hacia el este y oeste del valle, en donde prevalecían condiciones ecológicas similares (Figura 2) 


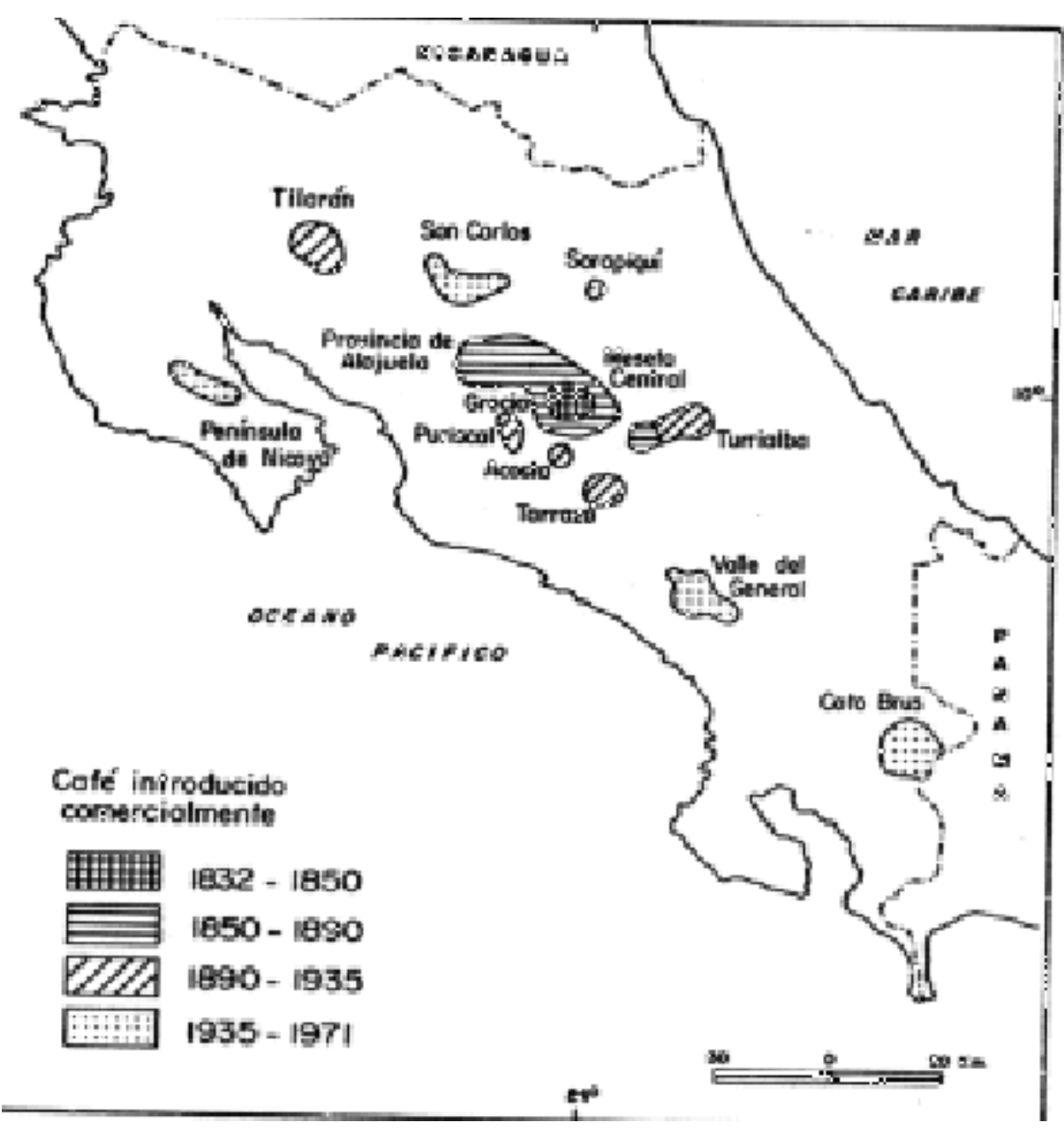

Entre 1850 y 1935 la expansión territorial del cultivo continuó, abarcando más tierras dentro del Valle Central y fuera de él (Tilarán, Puriscal, Acosta y Tarrazù). Después de 1935 y hasta 1962 nuevas áreas son incorporadas a la producción cafetalera en Coto Brus, San Carlos, Sarapiquí y la Península de Nicoya. (Hal: 1991: 16). En 1962 Costa Rica suscribe el Convenio Internacional del Café. Uno de los fines del convenio era detener la drástica caída de precios que se vivía desde la cosecha de 1957-1958. en aras de estabilizar los precios, el convenio llamaba a contener el crecimiento del área sembrada. En consecuencia con lo pactado, la superficie del sembrada del grano en Costa Rica permaneció estacionaria ente 1963 y 1973 (81.336 hectáreas en 1963 y 83. 406 en 1973). Después de este último año se da un crecimiento mesurado del área cultivada de modo que, en 1983, se sembraron 90.181 hectáreas (ICAFE: 1992: 3-4) aunque no existe información fidedigna sobre la evolución posterior de la extensión sembrada, datos 
recientes de venta de semilla indican que la expansión cafetalera continuó hasta 1986, para caer sostenidamente desde entonces (ICAFE: 1993: 61)

A pesar de la incursión de la caficultura en varias regiones del territorio costarricense a lo largo del siglo XX, el Valle Central continuó siendo la región cafetalera por excelencia del país, tanto por la superficie cultivada, como por el volumen y la calidad de las cosechas. El Valle constituyó el punto de propagación del cultivo, el centro de experimentación de los sistemas agrícolas cafetaleros, y la zona donde el impacto ambiental de la actividad ha sido mas violento.

La difusión espacial del café, relevante como es, expresa sólo una de las facetas de su evolución. Igualmente importante es examinar los sistemas bajo los cuales el grano ha sido producido.

\section{3-1850: LA BUSQUEDA DE UN MODELO AGRO-CAFETALERO}

Esta fase atañe a los albores de la caficultura, en las inmediaciones de la ciudad de San José y, de los otros asentamientos Principales del Valle, hay tres hechos de principal importancia para la comprensión del impacto tempranero del café. Primero, está el hecho de que el grano fue originalmente sembrado en tierras que habían sido previamente deforestadas. En efecto, como Hall (1991: 28) ha señalado, la vegetación predominante, hasta una distancia de 10 kilómetros de las ciudades principales del Valle, eran los pastos. Este era en caso de las cercanías de San José, donde, además de los pastos, existían importantes extensiones cultivadas de tabaco y alimentos. Aunque el establecimiento de San José databa de tiempos muy recientes, la deforestación había alcanzado niveles alarmantes. Tanto así que, en ocasiones, la municipalidad de san José se vio obligada a lanzar campañas de reforestación, a restringir la extracción de madera del potrero colonial de Las Pavas y a proteger los pocos remanentes de bosque que sobrevivían en otras áreas coadyacentes. Testimonio de la escasez de madera y leña fue la constante invasión, por otra parte los josefinos, de los bosques comunales de las localidades vecinas particularmente de los Tres Ríos ${ }^{1}$.

En un primer momento, así el café constituyó un medio de reforestación, antes que de deforestación. Pero se trató de una reforestación relativa, porque inicialmente los 
cafetos se cultivaron en bajas densidades, y porque no se usaron árboles de sombra, verdaderos reguladores de la insolación del cafetal, que habrían de popularizarse poco tiempo después. Como las plantas de café no toleran mucha competencia, es probable que los suelos "limpios", sembrados con una baja densidad de cafetos, y desprovistos de un estrato arbóreo, hayan sido más propensos a la erosión. Pero, dada la corta extensión cultivada, y dado también que los primeros cafetales se ubicaron en suelos de suaves pendientes, los efectos debieron ser leves.

La parte industrial del proceso, el beneficiado del grano, se llevó a cabo originalmente con el llamado "método seco" (secado del grano al sol en los patios de beneficio), y a muy pequeña escala. Esto permitió mantener la pureza de los ríos y quebradas en las cercanías de las plantaciones, porque ni la separación de la pulpa externa del café se realizaba por la fuerza de las aguas, ni los desperdicios del proceso iban a parar a las corrientes. Asimismo, los residuos eran esparcidos a lo largo de toda la geografía cafetalera, en la mayoría de las pequeñas fincas, en donde, generalmente, eran reutilizados como abono natural.

\section{0-1950: CONSOLIDACION Y DIFUSION DEL PRIEMR MODELO AGRO-CAFETALERO}

Hasta 1850, el desarrollo del café en Costa Rica había conocido una fase mas bien experimental. El café producido era destinado al autoconsumo y los métodos de labranza y beneficiado vivían su fase de ensayo y error. Los cafetales tenían una vocación doméstica y el beneficiado era realizado en las unidades familiares mismas, sin más recursos que la energía del sol y el trabajo familiar (Hall: 1992:49). A partir de 1850, aproximadamente, las cosas cambian de manera sustancial. Las exportaciones del grano se aceleran, lo que acarrea una radical reestructuración de la economía costarricense, que en adelante se orientará más y más al mercado internacional. De mayores alcances para nuestra discusión, hacia 1850 se perfila con claridad un sistema de cultivo y beneficiado del grano, de trascendentales secuelas ambientales.

En esta fase, las ya deforestadas tierras de los alrededores de San José y las otras

ciudades y pueblos principales, tierras comunales conocidas como de "La Legua", así 
como las localizadas a orillas del camino Real, continúan poblándose de cafetales. Sin embargo desde 1840 la propagación del café también territorios cubiertos de bosque, sobre todo hacia el oeste del Valle, en el área que se extiende entre Alajuela y San Ramón (Figura 2) Pocos decenios más tarde, hacia 1890,una activa colonización agrícola despunta en el extremo este del Valle, en la zona de Turrialba. Desde 1840, el próspero negocio cafetalero induce a una deforestación, cuyas consecuencias debieron haberse hecho sentir. El modelo agro cafetalero que emerge, no obstante, lleva a suponer que ese impacto fue modesto.

Después de los ensayos iniciales, para 1850 había cobrado forma un sistema de cultivo, que habría de perdurar durante los cien años siguientes. Nos referimos al sistema de café con sombra que, a pesar de afectar el medio, tendía en cierto grado a reproducir la arquitectura del bosque tropical. (Figura 3) 


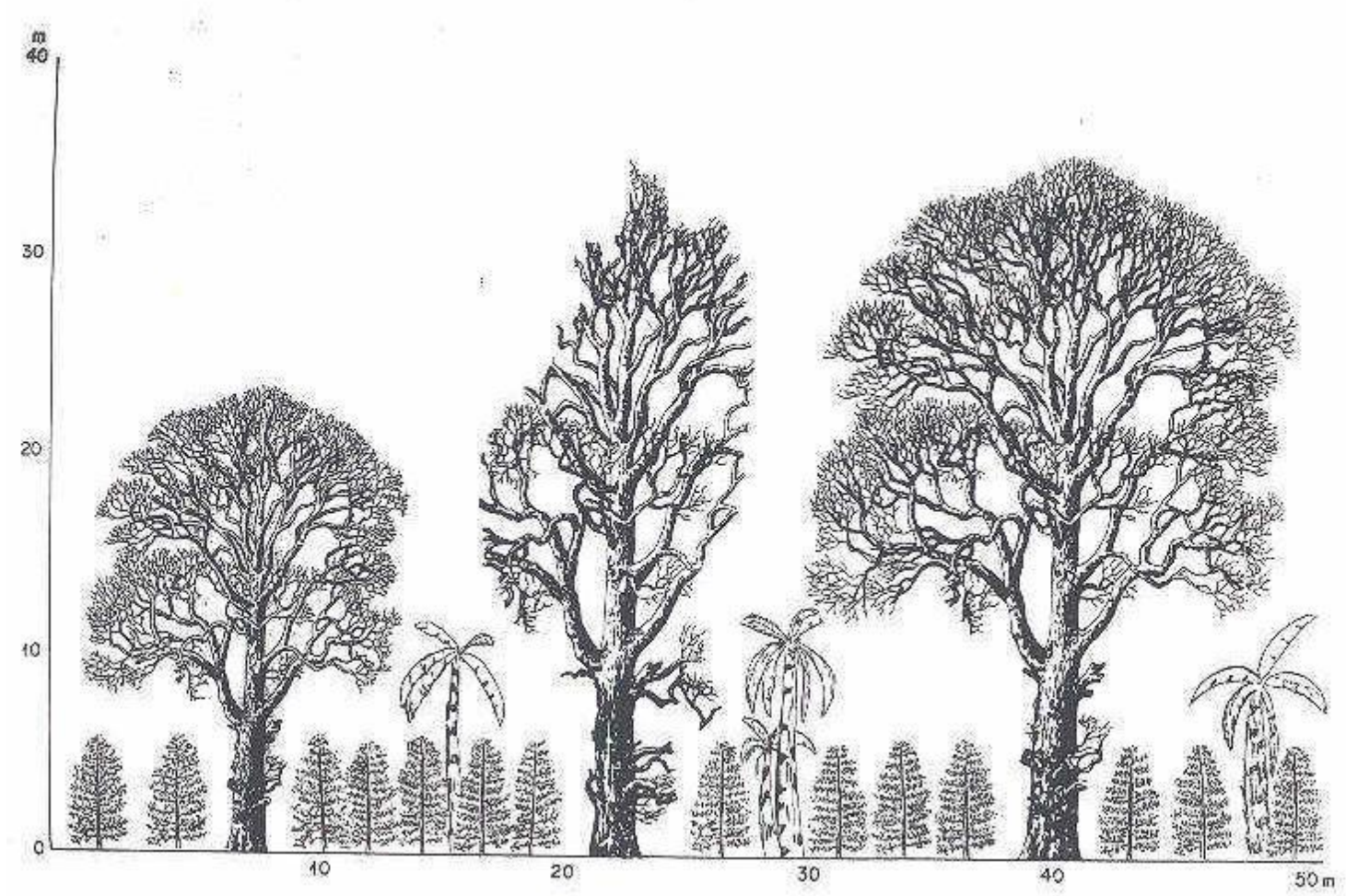

La agregación de árboles de sombra, sobre todo frutales como la guaba (Inga $s p$ ), el banano (Musa cuoudeshi), el guineo (Musa sapietum), el plátano (Musa paradisiaca), y el cuajiniquil (Inga sp.), o árboles como el poró (Eritrina sp.), cumple una importante misión como reguladora del agua en el ambiente. Los árboles interceptan una buena cantidad de la precipitación, protegiendo los suelos de los golpes directos de lluvia, y aminorando la erosión. La hojarasca liberada por este estrato protector, depositada en el suelo, además, limita el potencial erosivo de la lluvia que choca contra el suelo. Mucha del agua retenida por el piso arbóreo baja lentamente al suelo, mermándose la escorrentía superficial. Esto facilita la labor de evacuación de aguas de los ríos y quebradas, permite la infiltración de la humedad en el suelo y la alimentación de acuíferos, a la vez que se evita el desgaste físico de los suelos.

Además del crucial papel en la administración del ciclo del agua, los árboles contribuyen de otros modos en el funcionamiento del sistema. Regulan, por ejemplo, la actividad fotosintética y controlan la acción del viento. La hojarasca, depositada por el café mismo y las plantas y árboles de sombra, aporta nutrientes al suelo, reduciendo la 
necesidad de fertilizantes. De igual modo la presencia de hojarasca reduce el crecimiento de malezas, haciéndose menos necesario su combate con otros instrumentos. Una vegetación mas diversa, finalmente, da cabida a una fauna mas variada, que a su vez incide en los procesos físicos y químico del suelo para una análisis detallado de este agrosistema cafetalero ver Fournier: 1980)

En 1935 habían unas 46.677 hectáreas sembradas de café. El trabajo era el principal insumo de la caficultura, pues el uso de agroquímicos era raro: mas de dos tercio de las tierras cafetaleras no recibían fertilización ni tecnología química alguna, y las fincas que utilizaban agroquímicos lo hacían a muy baja escala. La variedad tradicional de café, conocida como Typica, predominaba abrumadoramente, y se sembraba en bajas densidades (1525 cafetos por hectárea, contra 3365 en 1981) (Aguilar et al: 1982:1-29 Aguilar, J. Barboza, C. y León, J. El desarrollo Tecnológico del Café en Costa Rica, CONICIT, San José). Las tierras, así sembradas presentaban una baja productividad, (2.6 toneladas métricas por hectárea, contra por ejemplo, un rendimiento de 7.2 toneladas en 1981)). La baje densidad del cultivo, los limitados insumos y tecnología aplicados y las debilidades productivas de la clase Typica, contribuían a este efecto. El otro extremo del argumento debe señalarse que los costos de producción eran bajos y que el cafetal producía cantidades considerables de frutas, madera, leña, y otros artículos que complementaban la economía campesina. Más significativo es que la imitación que el cafetal tradicional hacia del bosque, al aceptar la existencia de un estrato arbóreo, otro arbustivo, y una diversidad de especies de flora y fauna, tenía sin duda importantes ventajas ambientales. Tanto mejor era el sistema habida cuenta de que, hasta 1935, el café había cubierto tierras de suaves declives y drenaje fluido, por lo que los suelos habilitados no eran tan proclives a la erosión.

Otro aspecto que sin duda modificó el impacto ambiental de la caficultura, esta vez con consecuencias negativas, fue el sistema de beneficiado. Ya para 1850 se había afianzado el método húmedo de beneficiado (Hall: 1992: 49). Esta técnica, que usa el agua de ríos y quebradas para separar físicamente la cáscara del grano del café, contamina las aguas de dos formas. Primero, les carga de residuos, las llamadas "mieles" del café. Segundo, las cáscaras son lanzadas con una creciente frecuencia a los cauces de los ríos, convirtiéndose en un fuerte factor de contaminación, dado el incremento en la 
Demanda Biológica de Oxígeno (DBO). Este tipo de impacto, de vastas proporciones en el presente, era ya serio a principios de siglo (Boyce et al.1993a: 7-8).

Hasta el año 1935 el modelo caficultor, establecido en la segunda, mitad del siglo anterior, subsistió, sin mayores cambios. El sistema de siembra del grano permaneció inalterado, en tanto que el beneficiado húmedo fue objeto de un constante mejoramiento tecnológico que, sin embargo, no modificaba cualitativamente sus derivaciones ambientales.

Una relación del año 1939, del distrito cafetalero de la Uruca (Amerling: 1939), adyacente a la ciudad de San José y de gran tradición cafetalera muestra la persistencia de los sistemas tradicionales. Había en el distrito un total de veinte fincas, en todas las cuales se cosechaba café. El área cafetalera era de 873 manzanas, unas 603 hectáreas. Se producían en el distrito 12.279 fanegas, unas 580.5 toneladas métricas, lo que arrojaba un promedio ligeramente inferior a la tonelada métrica por hectárea, bastante bajo en comparación con épocas posteriores. El grueso de las plantaciones eran viejas: un 20\% de ellas tenían más de treinta años, y casi la mitad más de 15 años. Las plantas de café, todas de la variedad Typica, eran de porte alto, promediando entre 1.75 y 2.25 metros de altura. Los cafetos se sembraban en cantidades superiores a las conocidas en la época: el promedio nacional en 1953, era de 1.525 matas por hectárea. En la Uruca. En cambio, se plantaban hasta 2857 cafetos por hectárea, en los cafetales viejos, y unos 1374 en los cafetales más jóvenes. Diseminados en el cafetal o en las orillas del mismo, actuando como sombra o tapaviento se encontraban, entre otros, árboles y plantas de guineos, guaba, cuajiniquil, madero negro, poró, grevilia (Grevillea robusta Cunn). La presencia de estas especies era bastante significativa. La grevilia, se usada como sombra, crecía a razón de 143 árboles por hectárea; el poró, la guaba y el cuajiniquil se daban en un número promedio de 164 árboles por hectárea.

Los rendimientos eran, de acuerdo al informante, superiores a la medida nacional. Esto probablemente se debía que la mayor parte de las tierras eran abonadas. La materia orgánica que generaba el cafetal era el principal fertilizante. Pero además se abonaba con los sedimentos que acarreaban las quebradas que discurrían por el área y que eran atrapados en tanques de captación. En un sólo tanque se capturaban 400 toneladas de sedimentos en ocho meses, que eran aplicados durante la estación seca. Siendo el distrito 
de la Uruca de los mas avanzados, también conocía el uso de abonos químicos. El lodo de los ríos, empero, se reportaba como el de mejores resultados (Amerling: 1939). El aumento de la producción entre 1850 y 1950 fue, con ligeros altibajos, constante, pero se debió al acrecentamiento regular de las zonas cultivadas y no a cambios en las técnicas de producción. Con todo, el área cultivada de café hacia 1935 no sobrepasaba el 1\% del territorio nacional y estaba circunscrita casi enteramente al Valle Central. Entre 1935 y 1950 la difusión espacial del cultivo persiste, ahora para incluir territorios allende los límites del Valle (figura 2). Pero, a la vez, que se promovió la extensión del cultivo a nuevas tierras, se advierte, que la productividad del café costarricense es una de las más bajas del continente, y que la solución no puede provenir de la mera ampliación del área cultivada.

\section{0-1980: LA BUSQUEDA DE UN NUEVO MODELO CAFICULTOR}

En 1950 el área de labores era ligeramente superior a la de 1935 (48.885 hectáreas). El grano se producía a la usanza tradicional: prevalecía el uso de sombra en

los cafetales, los controles naturales de las malezas, el exiguo uso de abonos (sólo un $31 \%$ de las tierras recibían agroquímicos o abonos naturales), un bajo número de plantas de café por hectárea (1575) y la variedad de café Typica (Aguilar et al.: 1982: 1-29). Entre 1935 y 1950 se registra un descenso en la cosecha (de 2.6 a 2.4 toneladas por hectárea), asociada primariamente al envejecimiento de los cafetales, algunos de los cuales habían estado en explotación hasta por un siglo. El síntoma mas claro de este descenso en la productividad es el hecho de que, en 1950, muchos de los beneficios habían reducido el volumen de trabajo a la mitad de su capacidad.

Durante la década de 1950 se viven notables transformaciones en la producción cafetalera, de incalculables alcances ambientales. Entre otras cosas, se desarrollan nuevas variedades de café, se produce una renovación de cafetales, se generaliza el uso de abonos, herbicidas, plaguicidas, etc, y se promueve la irrigación y el drenaje, (Hall: 1991: 159). 
Un primer hecho es la adopción de híbridos mejorados de café, que producen mejores resultados. Así, si en 1950 la variedad Typica prácticamente no conocía competencia, treinta años después sólo cubría un tercio de las plantaciones del país (Aguilar et al: 1982: 1-34). Durante los 1960 se empezó a sembrar la variedad caturra , y en la década siguiente la catuai. Estas variedades difieren notablemente de la Typica, no sólo por su mayor productividad por planta, si no por su menor tamaño, lo que posibilita cultivarlas en mas elevadas densidades. A ello se debe que el número de cafetos por hectárea pasara de 1575, en 1950, a 3365, en 1981 (Aguilar et al: 1982: 1-29).

La difusión de estas nuevas formas fue el elemento desencadenante de permutas sustantivas en las plantaciones. En primer lugar, se tradujo en una reducción de la sombra, dado que se requiere de una mayor cantidad de energía solar para que una creciente cantidad de plantas en el cafetal. Los nuevos cultivares además, contrariamente a la Typica, presentan una fuerte resistencia al viento, lo que posibilita la eliminación de los tapavientos. En conjunto, hemos presencia una progresiva e inexorable reducción de árboles en las fincas de café.

En segundo, las demandas nutricionales de una dilatada población de cafetos aunada a la deforestación del cafetal, han estimulado un mayor gasto en fertilizantes químicos. La eliminación de las malezas, a su vez, que antes era una buena medida realizada por la vegetación alta de las plantaciones, debe ahora ser realizada por herbicidas artificiales. El rubro de los "materiales", entre los que sobresalen los agroquímicos, representaba sólo un 8\% de los gastos de las fincas, en 1953. Para 1980 estos desembolsos se habían elevado al 22.95\% (Aguilar et al: 1982: 4-20).

Desde 1950, al calor de las tendencias predominantes en el mundo occidental, Costa Rica paulatinamente ingresa a la era de la "Revolución Verde". No sólo el país se incorporó al movimiento, sino que fue pionero mundial, conjuntamente con Kenya, en la revolución verde del café (Benoid: 1993: 6). Los resultados como lo muestra la figura 4, no se hicieron esperar. Figura 4 INSERTAR FIGURA

La producción cafetalera costarricense se disparó de 462.896 fanegas, en 1953, a 2624176, en 1980. Antes que aún agrandamiento del área sembrada, los índices de producción se asocian a un uso mas intensivo de agroquímicos: en precios de 1966, el monto de agroquímicos pasa de 143 a 883 colones por hectárea entre 1953 y 1980. 


\section{EL IMPACTO AMBIENTAL DE LA CAFICULTURA CONTEMPORÁNEA}

Mas que de simples mutaciones técnicas, lo que ha acontecido es una redefinición del sistema agrocafetalero en su totalidad. El rasgo primordial del nuevos sistema de cultivo es la preponderancia de variedades de café cada vez menos tolerantes a la sombra. En el cafetal sin sombra, en contraste con la experiencia tradicional, los desajustes ecológicos se tornan mas agudos. El impacto ambiental de la caficultura reciente puede resumirse diciendo que el nuevo agrosistema:

a) reclama de la tierra cantidades excesivas de unos pocos nutrientes, que esta no es capaz de dar. Esto, sumado a los limitados aportes de humus que una vegetación escasa y unidimensional puede ofrecer, lleva a un empobrecimiento del suelo, e incita a un mayor uso de fertilizantes artificiales;

b) entraña una mayor aplicación de sustancias tóxicas para la lucha contra las pestes, que antes eran en gran medida controladas por la biodiversidad,

c) precisa de grandes inversiones en herbicidas químicos, para eliminar formas vegetales que rivalizan con la especie cultivada,

d) supone obras de infraestructura para informar las condiciones del cultivo (por ejemplo, drenar las tierras demasiado húmedas o irrigar las muy secas)

e) elimina el hábitat de centenares de especies, animales y vegetales, que se ven obligadas a migrar,

f) desprovee el suelo de una fracción cuantiosa de la capa vegetal, exponiéndolo a la erosión hídrica.

g) todo lo anterior que podríamos denominar transformación in situ, repercute en el ambiente externo: los ríos arrastraran aguas cargadas de sedimentos e impurezas químicas y las depositarán aguas abajo, a la vez que verán reducida o suprimida la capacidad para sustentar vida en sus aguas; las

especies desplazadas invadirán otros ambientes, planteándose problemas de competencia territorial y afectándose el dinamismo de poblaciones autóctonas. 
Las investigaciones destinadas a medir el efecto real sobre el ambiente de las nuevas modalidades de cultivo de café son todavía pocas, y no se conocen intentos de estimar el resultado conjunto de las distintas causas individuales. No existen, por ejemplo, estudios de naturaleza regional, que indaguen, en contextos específicos, el daño total causado al ambiente por los diferentes procesos y agentes pertubadores. Varios de estos vectores, no obstante, han sido analizados separadamente y permiten dibujar un panorama de las complicaciones que la nueva caficultura ha engendrado.

\section{EROSIÓN DE SUELOS}

Un interesante análisis experimental fue realizado en 1980 por Bermúdez, que pone de manifiesto los distintos niveles de erosión hídrica del suelo en cafetales con y sin sombra (Bermúdez: 1980). El estudio, fundamentado en la observación en parcelas experimentales, contrasta la situación de cafetales con sombra de poró y cafetales sin sombra. Del trabajo de Bermúdez se desprende que las pérdidas de suelo en los cafetales sin sombra son de $360 \%$ a $620 \%$ mayores que en las plantaciones arboladas. El volumen de sedimentos en la escorrentía superficial que emana de estos cafetales después de un aguacero es tan alto que inutiliza las aguas para el consumo humano o comercial. El desgaste de los suelos - concluye el reporte- malgasta los nutrientes e implica una mayor aplicación de fertilizantes. Además, se determino que en el cafetal a plena luz la invasión de malezas es muy elevada lo que multiplica los costos del control de hierbas (48-52). En el cafetal con sombra de poró, en cambio, la disminución de la erosión es notoria: se consigue una reducción de casi un $85 \%$ de la erosión registrada en las parcelas de monocultivo. Asimismo, el control natural de las malezas es mas eficaz, y la necesidad de fertilizantes moderada. En conjunto, Bermúdez concluye que el sistema agroforestal constituye una efectiva práctica de conservación de suelos, y que la eliminación de la sombra es un factor primordial de erosión (Bermúdez:1980: 52).

Sancho (1991), en un línea similar de análisis, demostró la desigualdad perdida de suelos en cafetales, de acuerdo al tipo de manejo de malezas y la cobertura vegetal presentes en ellos. El autor determinó que la erosión es más alta en los cafetales en que el café no es acompañado por ningún otro tipo de cobertura vegetal. El segundo lugar en 
erosión lo ocupan los suelos en que se practica el control químico de malezas, en tanto que la erosión se redujo drásticamente en aquellos cafetales en que se intercalaba plantas de Arachis pintoi, o en que se practicaba la "chapia", dejando el residuo sobre el terreno. Los niveles de pérdida de nutrimentos y materia orgánica, igualmente, variaban en asocio a los sistemas de manejo de las malezas y la cobertura (Sancho: 1991: 105-108).

Visto en una dimensión más espacial, debe acotarse que la erosión inducida por el nuevo esquema agrocafetalero debe haberse agudizado, a la luz de las transformaciones que recientemente se han operado en las principales zonas productoras del café de Costa Rica. nos referimos a la expansión masiva del área metropolitana de San José. El proceso de urbanización que hasta 1950 había sido tenue, adquiere gigantescas proporciones desde entonces. Particularmente importante para los efectos de esta discusión es destacar que la formación de una gran área metropolitana se da a expensas de muchas de las mejores tierras cafetaleras del país. La conversión de suelos agrícolas en urbanos, que entre 1924 y 1945 fue de sólo 13.3 hectáreas anuales, se elevó a 95.9 hectáreas entre este último año y 1957, a 170.4 hectáreas entre 1957 y 1965, y a 695.9 hectáreas anuales entre 1965 y 1980 (Vargas: 1985: 349). La ocupación del suelo agrícola por espacios residenciales, en las tierras más planas, ha provocado la migración del café a las laderas, en donde las pendientes mas acentuadas, en combinación con la deforestación del cafetal, son responsables por una mayor proclividad a la erosión.

\section{CONTAMINACION DEL SUELO Y DE LOS FRUTOS DEL CAFE}

La poca investigación realizada en la materia denota que, como era presumible, tanto los suelos como las bayas del café resienten hoy día el uso de los agroquímicos. Por ejemplo, la investigación de Rojas (1984) en suelos de tres zonas cafetaleras del valle Central (Atirro, en el este del valle, Barreal, en la parte central, El Rosario y Naranjo, en el oeste) demostró la permanencia de Paraquat en el suelo. Paraquat es un tipo de herbicida, destinado al control de malezas, de uso en los cafetales costarricenses desde $1960 .^{2}$ En las fincas estudiadas por Rojas, el Paraquat había sido empleado dos veces por 
años durante los últimos dos decenios. A pesar de que el herbicida se aplica al follaje de las malezas, Rojas encontró considerables residuos de Paraquat en el suelo, incluso en el caso de Atirro, donde no se había usado en los últimos tres años. La investigación concluye que el Paraquat no se degrada rápidamente, y que es factible que una fracción del químico sea absorbida por las raíces de las plantas y trasladada a los frutos (Rojas: 1984: 54).

En el combate de enfermedades del café como el "ojo de gallo" (Miscena citrocolor), "enfermedad rosada" (Cortisium salmonicolor), y "mal de hierba" (Pellicularia koleroga) se ha recurrido al arseniato de plomo, un cancerígeno probado. Vestigios de esta sustancia también han sido detectados en los suelos cafetaleros del país, así como en las hojas y las bayas del café (Hilje et el: 1987: 55).

\section{LA CONTAMINACIÓN DE LAS AGUAS SUPERFICIALES}

La descarga de materia orgánica en los ríos es la principal fuente de contaminación de agua en Costa Rica. De todo el material orgánico lanzado a los cursos de agua superficial, un $80 \%$ proviene del Valle Central, y es transportado, sobre todo, por el río Grande de Tárcoles (Ramírez y Maldonado: 1991: 97). El beneficiado húmedo del café, por su parte, es el principal responsable del excesivo volumen de materia orgánica en las aguas de los ríos. La magnitud de este impacto puede comprenderse mejor si se considera que el beneficio del café, por si sólo, aporta más contaminación al Tárcoles que toda la Gran Área Metropolitana de San José, en la que reside mas de un millón de

personas. En la época del beneficiado, el río Tárcoles acarrea en sus aguas una carga orgánica equivalente a la producida por unos seis millones de personas, el doble de la población entera del país (Incer. 1992: 2).

Varias de las cuencas mayores del país, entre ellas la del Reventazón, el Grande de Térraba, el Parrita y el de San Carlos, reciben aguas del beneficiado del café. El impacto es muchísimo mas elevado en la cuenca del Tárcoles, a la que el café aporta un $68 \%$ de toda la carga orgánica, contra sólo un 14\% producido por las aguas negras domésticas de más de un millón de habitantes y un 18\% de origen industrial (Ramírez y Maldonado: 1991: 101). 
La contaminación de varios ríos del país, tiene variadas repercusiones ambientales. No se trata sólo del efecto inmediato en la flora y fauna fluvial, hoy prácticamente desaparecidas de las putrefactas aguas del Tárcoles. Muchos ríos del Valle Central, al recibir sustancias nutritivas del beneficiando y la agricultura, desarrolla poblaciones exageradas de lirios y algas, que a su vez actúan como fijadoras de sedimentos, incentivando la sedimentación en represas hidroeléctricas. El efecto de las contaminadas aguas de los ríos se hacen sentir en las zonas donde son usadas en la irrigación para la agricultura, en el litoral, etc., como adelante se discute.

\section{CONTAMINACIÓN DE AGUAS SUBTERRANEAS}

La configuración climática, geológica y geomorfológica del valle central ha permitido el desarrollo de grandes acuíferos. La mezcla de precipitaciones elevadas entre los meses de mayo y noviembre la permeabilidad de los suelos volcánicos, así como la inclinación del relieve, permiten la percolación de mucha del agua de lluvia y la formación de mantos subterráneos. La importancia de estos acuíferos es cada día mayor, dada la demanda de agua derivada de un crecimiento urbano explosivo, y vista la contaminación de las aguas superficiales. Siendo el consumo de agua superior año tras año, y resultando cada vez más oneroso el tratamiento de las aguas superficiales, por sus grados de contaminación, no es de extrañar que las aguas subterráneas se hayan venido constituyendo en una salida limpia y barata a la situación. Actualmente se succionan unos 2300 litros por segundo de agua subterránea, de los cuales se surte un $60 \%$ de los habitantes de San José, Heredia y Alajuela, las tres ciudades mas importantes del país, amen de una cantidad considerable de industrias (Reynolds: 1991:114) ${ }^{3}$.

Reynolds (1991), analizando la cuenca del río Virilla, una de las tres componentes de la cuenca del río Tárcoles y precisamente en la que se localizan los principales acuíferos, han revelado la presencia de cuantiosas cantidades de nitratos en las aguas subterráneas. Estos nitratos, mundialmente ligados al desarrollo agropecuario y al uso de tanques sépticos, han sido relacionados con diversas enfermedades, algunas de ellas mortales, y responsabilizados como probables productores de cáncer. 
En la cuenca del río Virilla, los nitratos no introducidos por los mecanismos de fijación atmosférica provienen de la producción y el beneficiado del café, y de los tanques sépticos en los cafetales, los nitratos ingresan al sistema por medio de la fertilización. En promedio, se aplican 270 kilogramos de fertilizantes de nitrógeno por hectárea al año. Existe una propensión a un uso mayor de este tipo de abono, dado que en el área se ha generalizado el café sin sombra, que precisa de una mayor fertilización artificial, y porque se ha propiciado el uso de variedades de café que responden mejor a la fertilización con nitrógeno (caturra y catuai). Parte de los nitratos son absorbidos por los cafetos, otro tanto es retenido en el suelo o enviado a la atmósfera, pero una porción considerable desciende hasta los acuíferos, sobre todo durante la estación lluviosa. (Reynolds:; 1991: 113).

La investigación de Reynolds estableció dos tipos de correlaciones significativas. Primero determinó que la cantidad de nitratos en las aguas subterráneas coincide con los meses de la estación lluviosa, que es el momento en que los nitratos son arrastrados por el agua a los acuíferos. Segundo, evidenció una estrecha relación entre uso del suelo y presencia de nitratos: en las zonas altas de la cuenca (más de 1600 de altitud) en las que imperan el bosque(14.5\%) y los pastos (80.5\%), ninguno de los cuales es fertilizado químicamente, la concentración de nitratos en el agua subterránea es baja $(0.37 \mathrm{ug} / \mathrm{ml})$. En las tierras intermedias (1000-1500 metros), en las que el café acapara un 73\% del suelo, los valores escalan a $1.93 \mathrm{ug} / \mathrm{ml}$. En las partes bajas de la cuenca, donde predominan el casco urbano (47\%) y el café (34\%) la concentración de nitratos es todavía más alta $(4.45 \mathrm{ug} / \mathrm{ml})$, como resultado de las deposiciones de una infinidad de tanques sépticos, del café en el área, y de los procesos originados en las alturas del valle.

En las partes bajas de las cuencas del Virilla, los índices de nitrato en las aguas subterráneas se aproximan temerariamente, e incluso sobrepasan, los montos recomendados por la Organización Mundial de la Salud (10 ug/ml). Los peligros para la salud son inminentes, porque estas aguas se consideran puras y se suministran al publico sin los tratamientos que corresponderían al caso (Reynolds: 1991: 4-112)

\section{LA IRRIGACION CON AGUAS CONTAMINADAS}


En el Valle Central, las contaminadas aguas de varios ríos y riachuelos son usadas para la irrigación. Hace pocos años Salas (1987) condujo una evaluación de los efectos de las aguas contaminadas en la producción agropecuaria de la zona de Belén, ubicada entre el río Bermúdez y la Quebrada Seca. Las aguas de estas dos corrientes son utilizadas durante la estación seca, para la producción de hortalizas, granos básicos y verduras. Las consecuencias del regadío con aguas contaminadas resultaron elocuentes: brotes de enfermedades fungosas o enfermedades fisiológicas en las plantas mismas, contaminación del suelo, deterioro de equipos por el efecto corrosivo de las aguas sórdidas, etc. En general, se reportaron cuantiosas mermas de la productividad y aumentos en los costos de producción (Salas: 1987).

\section{PROBLEMAS DE SALUD}

Las consecuencias sanitarias del uso y abuso de agroquímicos han sido una preocupación mundial durante décadas recientes, por lo que no es del caso extenderse en la materia. Se sabe que muchas de estas sustancias son capaces de producir la afección y muerte de las personas expuestas, directa o indirectamente, a los agroquímicos, e incluso en sus descendientes. En la euforia de la "revolución verde", la agricultura costarricense, como se vio, a apelado intensivamente al uso de agroquímicos. En 1987 se reportaba que el consumo de agroquímicos llegaba a 4.6kg por persona, cifra que superaba con creces, por ejemplo, la de los Estados Unidos, en donde sólo se aplica $2 \mathrm{~kg}$ per capita (Hilje et al: 1987: 47). No se conoce con exactitud los alcances del problema en Costa Rica, pero se supone que los daños derivados de los agroquímicos deben ser severos, por que en el país comúnmente se abusa de su uso (Hilje et al: 1987), y porque a menudo se aplican sustancias que han sido prohibidas o restringidas en otros países (Ramírez y Maldonado: 1988). Véase, a manera de ejemplo, la participación de Paraquat, típico de la caficultura, en el cuadro de defunciones originadas por plaguicidas (Figura 6). 


\begin{tabular}{|l|l|l|l|l|l|l|l|l|}
\hline \multicolumn{2}{|l|}{ Figura 6 DEFUNCIONES CAUSDAS POR PLAGUICIDAS, 1980-1996 } \\
\hline $\begin{array}{l}\text { Grupo } \\
\text { Químico }\end{array}$ & 1980 & 1981 & 1982 & 1983 & 1984 & 1985 & 1986 & Total \\
\hline Paraquat & 22 & 19 & 18 & 33 & 25 & 27 & 17 & 161 \\
\hline Otros & 1 & 10 & 21 & 16 & 18 & 22 & 26 & 124 \\
\hline Total & 23 & 29 & 39 & 49 & 43 & 49 & 43 & 285 \\
\hline
\end{tabular}

Fuente: Hilje et al: 1987: 45

Unas 41 personas mueren cada año por envenenamiento con agroquímicos; de ellas, 23, en promedio, fallecen por intoxicación con Paraquat, el herbicida de los cafetales. Las defunciones, por supuesto, no son más que el pico del iceberg, pues sólo un pequeño segmento de los hospitalizados fallecen, y, lo que es peor, sólo una minoría de las personas intoxicadas acuden a los hospitales. Ña absorción gradual de pequeñas cantidades del químico de consecuencias en el largo plazo, generalmente pasa desapercibida (Hilje: 1987)

\section{LA CONTAMINACION DEL LITORAL}

Las aguas contaminadas de las regiones cafetaleras terminan su viaje en el mar, en donde tienen efectos todavía poco estudiados. El río Tárcoles, por ejemplo, el mas sucio del país, desemboca en la costa del Pacífico, y crea una tensión al menos en dos niveles. En primer lugar, contamina el patrimonio pesquero de las aguas cercanas a la costa, erigiéndose en un fuerte obstáculo para la pesca artesanal. En segundo lugar, produce la contaminación de las playas ubicadas entre su desembocadura y la ciudad de Puntarenas, una de las regiones turísticas de más raigambre en Costa Rica (Ramírez y Maldonado: 1991: 67-101)

\section{OTRAS REPERCUSIONES EN EL AMBIENTE}


Costa rica, no obstante su pionero sistema de áreas protegidas, exhibe una de las tasas de deforestación mas altas de América Latina. Entre 1950 y 1984 se derribaron 17032 kilómetros cuadrados, siendo al extensión total de Costa Rica 51100 kilómetros cuadrados. Para 1984 sólo un $18.9 \%$ del país estaba cubierto por bosque denso, y un $13.9 \%$ con bosques poco densos. Esto ha sucedido a pesar de que, de acuerdo a la capacidad de uso dela tierra, un 64.25 del territorio nacional debería estas cubierto de bosques (Ramírez y Maldonado: 1991: 59-61)

Esta reducción de la floresta, entre muchas otras cosas, ha representado una drástica disminución del hábitat de miles de especies animales y vegetales. El cafetal arbolado de antaño, en ese sentido, constituyó un importante albergue de centenares de especies, muchas de las cuales hoy se encuentran amenazadas o en peligro de extinción. La deforestación del cafetal, así como el uso indiscriminado de sustancias tóxicas, ha hecho aún más precaria la situación de numerosas especies. Aves como el gavilán (Leucopternis princeps), la lechuza (Otus guatemalae), el colibrí (Lophornis adorabilis), el pájaro carpintero (Melanerpes chrysauchen), pecho amarillo (Myodynastes hemichyrysus), o mamíferos como el murciélago (de la familia Soricidae), el conejo de monte (Sylvilagus floridanus), diversos tipos de ardillas (familia Sciuridae), etc, todos ellos amenazados (Ramírez y Maldonado: 1991: 128-134)), fueron residentes consuetudinarios de los cafetales.

Está finalmente, un impacto menos susceptible al ser expresado en cifras, pero no por ello menos importante: el cafetal tradicional, con su estrato arbóreo, su microclima, su diversidad de especies comestibles, su variada flora, en fin, su diverso mundo interior, represento una fuente de esparcimiento y un complemento de las economías hogareñas. Cualquiera que haya crecido entre cafetales sabe lo que esto vale y se percata del significado de este paisaje hoy ya casi totalmente desaparecido.

$* * * * *$

Como se mencionó, no se sabe de estudios destinados a evaluar el impacto integral de las distintas formas de contaminación producidas por el café. Pero evidentemente hay una concatenación, tanto a nivel de causas como de efectos. Si tomammos por caso la sección del Valle Central de más larga cronología cafetalera, en 
las proximidades de Heredia, Alajuela y San José, podríamos intentar un enfoque sistémico, que nos permitiera intentar una síntesis de procesos.

Hasta 1950, esta porción del valle revestida del antiquísimo cafetal arbolado, de un leve impacto ecológico. en medio de los cafetales, a una altitud media de 1200 metros, se ubicaba una red de pequeñas ciudades y pueblos. A partir de 1950 se verifican dos procesos. Por una parte, una urbanización explosiva reviste de cemento los mejores campos cafetaleros; las fértiles, ligeramente inclinadas y bien drenadas tierras del fondo del valle. Por otra, se constata una transformación del sistema agrocaficultor, para dar paso al monocultivo del café en altos rendimientos. Se asiste, entonces a un desplazamiento territorial del café a zonas de pendientes más acentuadas, a la vez que se reduce la cubierta vegetal.

De lo arriba descrito se infiere que los suelos son más susceptibles a la erosión, porque en presencia de un manto vegetal mermado, y un relieve de mayor inclinación. La acción erosiva del agua es más potente. La pérdida de nutrientes por la erosión, la especialización del cafetal, el florecimiento de más malezas y los requerimientos del mayor número d cafetos, suscita una creciente dependencia de los agroquímicos, muchos de ellos tóxicos, penetran las plantas y los frutos del café. Una buena porción se almacena en el suelo o es arrastrada a las aguas superficiales o subterráneas.

Como la agricultura se desplaza a los flancos del valle, la contaminación se inicia en las zonas mismas de captación de aguas: donde los ríos tienen suficiente caudal como para justificar su aprovechamiento, el agua ya está contaminada. En las partes altas de la cuenca, las aguas subterráneas, separadas de la superficie por un delgado estrato de porosos materiales, son también más propensas a la contaminación. Las aguas ahora se ensucian más y más temprano.

La siempre en aumento producción cafetalera, a su vez, ha multiplicado el efecto contaminante del beneficiado húmedo. El volumen de desechos progresa año con año, y con ellos los desordenes ambientales. El Tárcoles y sus tributarios, otrora fuentes de agua pura y recreación, pasan a ser el canal de escape de las aguas putrefactas que una urbanización sin control y la caficultura excretan. Estas aguas pestilente, usadas en la agricultura, son el mandadero de todas suerte de problemas; llegadas al mar, dan al traste 
con la pesca y el turismo, para no mencionar sus probables consecuencias en el delicado manglar del litoral o en otras especies de la costa.

Pero el precio lo pagan los que viven aguas abajo, en las riberas de los ríos o en la costa. Pero también lo pagan los residentes de las áreas de café. Los que trabajan corren mayor riesgo de enfermar, los que tienen cafetales han perdido una magnífica fuente de leña, madera y de variados alimentos de consumo familiar. Sus animales doméstico, si los tienen, no pueden vagar por el cafetal en busca de alimentos, so pena de perecer intoxicados. La población toda ha visto esfumarse el encanto del cafetal-bosque, víctima de la implacable lógica económica del cafetal de alta productividad.

\section{EL CAFE EN LA ENCRUCIJADA}

Que la caficultura vive en una de las peores vicisitudes de su historia es algo que nadie discute. Basta constatar que en agosto de 1992 los precios del grano cayeron al punto más bajo de los últimos 25 años, para percatarse de la gravedad de la situación. Tanto más serio se torna la cuestión, si se repara que más que una cuestión circunstancial, lo que se vive es una crisis combinada del modelo de producción y comercialización del grano. La revolución verde en el café, de la que Costa Rica constituyó el arquetipo, pero que se extendió a todos los países productores, ha llevado a un incremento constante de la oferta del grano, a una crisis de sobreproducción, que repercute en un descenso sostenido de los precios. El ocaso de la era de las cuotas del café y la instauración del "libre comercio" como principio de colocación de existencias, en 1989, ha dejado a los países productores a merced de las grandes firmas comercializadoras del grano, sin que mejore tampoco ala situación para los consumidores efectivos del café. La debacle de la producción cafetalera es contundente, y no se vislumbran salidas inmediatas al problema.

En Costa Rica, el efecto de la crisis asume dimensiones catastróficas, que ponen en tela de duda la perdurabilidad misma del sector cafetalero. La cosecha de 1988-1989 fue la mejor de la historia, y la de 1991-1992 la segunda mejor (ICAFE: 1993: 71). Entre 1988 y 1992 las exportaciones crecieron a una tasa anual del 6\% (Rojas: 1993: 6). Irónicamente, las tasas de ganancia han caído, al punto que en 1992 la actividad dejó saldos negativos. Las consecuencias no se han hecho esperar: muchos productores han 
sido eliminados. De los que subsisten, un porcentaje cada vez mayor no abona (93.5\% en 1990 contra 85.9 en 1991); se aminora la aplicación de herbicidas y fungicidas (ICAFE: 1993: 66, Rojas: 1993: 4). En fin, el modelo caficultor muestra síntomas inequívocos de agotamiento: desde el punto de vista económico el café se ah vuelto insostenible.

El café, hasta no hace mucho tiempo fue nervio de la economía costarricense, da cuenta hoy día de sólo un 1.1\% del Producto Interno Bruto, un paupérrimo $11.1 \%$ del valor de las exportaciones, un $0.1 \||| 5$ de los ingresos del gobierno y un lastimero $6 \%$ del empleo nacional (Rojas: 1993: 6-7).

A la vez que esto sucede, las externalidades del café son elocuentes: la caficultura actual es también ecológicamente insostenible. La fuerza de las circunstancias obliga a preguntarse hasta qué punto debe el país empecinarse en un modelo agrocafetalero que ya no es rentable para el productor y que ocasiona serios daños al ambiente, daños que inexorablemente terminarán engrosando las ya deficitarias cuentas nacionales.

El primer aspecto que amerita escrutinio es el precepto del incremento de la producción y la productividad como estrategia adaptativa del país en el mercado mundial cafetalero.

La figura 9 pone de manifiesto que no existe, en el caso costarricense una relación directamente proporcional entre la producción, la productividad y los beneficios generados por la actividad cafetalera. 


\begin{tabular}{|c|c|c|c|c|c|c|c|c|}
\hline \multicolumn{9}{|c|}{ Fig. 7 PRODUCCIÓN, PRODUCTIVIDAD Y GANAN CIAS CAFETALERAS, 1951-1980 } \\
\hline$\underline{\mathbf{A N} O}$ & \multicolumn{2}{|c|}{$\frac{\text { GANANCIAS POR Ha. }}{\text { (Colones de 1966) }}$} & \multicolumn{2}{|c|}{$\frac{\text { PRODUCCIÓN }}{\text { (Fanegas) }}$} & \multicolumn{2}{|c|}{$\frac{\text { PRODUCTIVIDAD }}{\text { (Fanegas/hectárea) }}$} & \multicolumn{2}{|c|}{$\frac{\text { PRECIO }}{\text { (dólares/saco) }}$} \\
\hline & & Incremento & & Incremento & & Incremento & $\$$ & Incremento \\
\hline 1951 & 2017 & & 384533 & & 7.9 & & 6.78 & \\
\hline 1968 & 1271 & -36.99 & 1605953 & 317.64 & 22.3 & 182.28 & 5.22 & -23.01 \\
\hline 1974 & 3018 & 137.45 & 1812591 & 12.87 & 20.4 & -8.52 & 9.78 & 87.36 \\
\hline 1977 & 6358 & 110.67 & 2025001 & 11.72 & 23.6 & 15.69 & 30.01 & 206.85 \\
\hline 1980 & 2136 & -66.40 & 2624176 & 29.59 & 29.4 & 24.58 & 41.38 & 37.89 \\
\hline
\end{tabular}

Fuentes: ICAFE: 1992: 30-31, Aguilar et al: 1982: 4-16. 
Entre 1951 y 1968 la producción se multiplicó en más de cuatro veces y la fertilidad casi se triplicó. Las ganancias por hectárea, sin embargo, cayeron, . entre 1968 y 1977, en cambio, los beneficios crecieron a un ritmo mayor que el de la producción y la productividad. De 1977 a 1980, como al inicio del período, los beneficios caen, a pesar de la creciente productividad cafetalera. Los datos de la Figura 7 ponen al descubierto un hecho, por lo demás, esperable; los precios en el mercado internacional, antes que la producción, la productividad por área o los costos en que se incurra, son los que establecen los niveles de ganancia. Y en este mercado Costa Rica, que produce un modesto $2.9 \%$ del café mundial exportable, tiene muy pocas posibilidades de incidir.

Desde el debut mismo de la caficultura en Costa Rica, se ha propendido al aumento constante de la producción. Hasta 1950, aproximadamente, la producción creció como resultado del ensanchamiento del área cultivada. Desde entonces, la superficie sembrada ha crecido lentamente, pero, gracias al adelanto técnico, los rendimientos por hectárea se han elevado sin cesar. Este mecanismo permitió a los cafetaleros sacar ventaja de las fases de bonanza o sobreponerse a los ciclos de caída de los precios. En la situación actual las cosas lucen diferentes, porque producir mucho puede significar perder mucho, en un mercado saturado. La pregunta ahora no es cuánto ganar sino cómo ganar; el asunto no es el incremento de las productividad, sino de las ganancias por hectárea. Productores grandes, como Brasil o Colombia, pueden tratar de modificar las condiciones del mercado. Países pequeños como Costa Rica, en cambio, deben trazar estrategias adaptativas para condiciones de precios que se definen externamente.

La salida para píses como Sota Rica podría estar en disminuir los costos, y no en continuar por el tortuoso camino del aumento de la cosecha; en producir menos, a bajo costo, y no más, a costos elevados. La reducción de costos, aún a expensas de cierto grado de productividad, traería muchas derivaciones saludables: llevaría a la sustitución de insumos importados con trabajo nacional; facilitaría la colocación de la producción del país en el mercado, porque es más factible vender menos; contribuiría en el largo plazo, mediante la restricción de la oferta, a la fijación de precios justos para el grano; daría más posibilidades al pequeño productor, al hacerlo más dependiente del trabajo familiar y menos de las costosas inversiones. Finalmente, y lo que es más relevante desde el punto de vista de este ensayo, limitaría muchísimo el impacto ambiental de la caficultura. 
Como puede adivinarse, se está abogando en este ensayo por el regreso al principio del cafetal con sombra, de una probada adaptación a las condiciones tropicales. No se trata, desde luego, de un retorno al esta de cosas de los mil novecientos treintas o cuarentas. De lo que se trata es de recuperar un modelo agrocafetalero, al que debería sumarse el conocimiento que muchos lustros de experiencia e investigación han permitido acumular. Hoy sabemos más que antes de abonos orgánicos, o del mejoramiento de las variedades de café, o del manejo de la sombra o de las variedades de café. Hoy conocemos de especies mas rentables, los cítricos, por ejemplo, que pueden funcionar como sombra del cafeto. Las densidades de cultivo pueden ser mayores que en el cafetal tradicional, sin menoscabo de la esencia misma del sistema. Las actitudes gerenciales han cambiado, y no hay razón para retroceder a los cánones patriarcales del pasada. En fin, mucha agua a discurrido bajo el puente como para preconizar una mera vuelta al pasado.

Acaso el denominado "café ecológico" sea la expresión mas acabada del principio defendido. La supresión completa de los agroquímicos traería de nuevo la limpieza al cafetal. El café orgánico ya se cultiva en Costa Rica y muchos otros países (Boyce et al: 1993 y 1993a). Es cierto que su mercado es todavía pequeño e incierto, y no representa una solución viable para el conjunto de los países cafetaleros. Pero también es verdad que un país como Costa Rica, que cosecha cantidades modestas de café, que recogería aún menos toneladas de café orgánico, y que ya no depende como antaño del café, podría apostarle mucho a esta línea de desarrollo. Los riesgos de una estrategia de esta naturaleza son numerosos, pero son contrarrestados por la seguridad de que el modelo vigente no sacará la caficultura nacional del atolladero. La certeza existe, eso si, de que las perjudiciales externalidades ambientales serán eliminadas. El estado costarricense, que es el que a fin de cuentas deberá limpiar las aguas y los suelos, curar a los enfermos y tratar de contener la fuga de divisas que los agroquímicos implican, debería apoyar esta penosa transición, como lo hizo en el pasado cuando se trató de incursionar en la revolución verde. 


\section{CONCLUSION}

Después de siglo y medio de agricultura del café, atestiguamos hoy día un peligroso marchitamiento de la caficultura. El café ya no es rentable. ¿Debería entonces abandonarse su cultivo?. Esto, a fin de cuentas, acabaría con los trastornos ecológicos creados por el café. Más allá de este simplismo, es necesario desatacar dos hechos. Primero, no hay ninguna razón para creer que el café será sustituido por actividades menos perniciosas. Muy por el contrario, los negocios que hoy se desarrollan, la producción de artículos agropecuarios no tradicionales, piñas, fresas o flores, intensifican las facetas negativas que la caficultura ha presentado. Del café sabemos, por el contrario, que es adaptable a la fisonomía del ambiente. Tampoco debe olvidarse que el café es ya parte del patrimonio cultural de la nación. Su extinción supondría una pérdida de incalculables consecuencias. ¿No es acaso cierto que la visión de la patria, que con tanto ahínco pregonan las costarricenses, tiene como emblema la pequeña propiedad cafetalera?

Más que una cínica renuncia a la caficultura, de lo que se trata es de reorientar la actividad. En esto en Costa Rica hay experiencia. Costa Rica introdujo por primera vez el cultivo comercial de la baya en Centroamérica, y fue el primer estado en hacer de la caficultura la llave para la salida del marasmo que siguió a la independencia, un siglo más tarde, Costa Rica se puso ala vanguardia mundial de un proceso que llevó a una expansión sin precedentes de la productividad cafetalera. No hay entonces base alguna para desconfiar de la capacidad del costarricense para hacer de la agricultura del café un negocio próspero y limpio, en el sentido más amplio de la palabra. 


\section{BIBLIOGRAFÍA}

Amaerling, R. 1939. "Estudio sobre las condiciones agrícolas del distrito de la Uruca." Trabajo inédito, San José.

Benoit, D. 1993. "La crisis del mercado cafetalero internacional en una perspectiva de largo plazo" Ponencia presentada en el Simposio Internacional “Modernización tecnológica, cambio social y crisis cafetalera". UNA-ICAFE, Heredia.

Bermúdez, M. 1980. "Erosión hídrica y escorrentía superficial en el sistema de café (Coffea arabica L.) poró (Erythrina poeppigiana, Walpers O.F.Cook) y laurel (Cordia ailiodora, R \& P. Cham.) en Turrialba, Costa Rica" Tesis de Maestría inédita, CATIE, Turrialba, Costa Rica.

Boyce, J. Fernádez, A., Fürst, E. y Segura, O. 1993. "Sustentabilidad de la producción cafetalera costarricense y conveniencia del café orgánico como alternativa" Trabajo inédito elaborado para la Maestría en política económica para Centroamérica y el Caribe. Universidad Nacional, Heredia.

Boyce, J. Fernádez, A., Fürst, E. y Segura, O. 1993a. "Crisis e innovación cafetalera en Costa Rica: El café orgánica como opción de desarrollo sostenible" Ponencia presentada en el Simposio Internacional "Modernización tecnológica, cambios social y crisis cafetalera". UNA-ICAFE, Heredia.

Fournier, L. 1980. "Fundamentos ecológicos del cultivo del café" Instituto Interamericano de Ciencias Agrícolas-Organización de Estados Americanos, San José.

Hall, C. 1991. "El café y el desarrollo histórico-geográfico de Costa Rica" Editorial de Costa Rica, San José.

Hilje, L.; Castillo, L.; Thrupp, L.; Weseling, H. 1987. “El uso de plaguicidas en Costa Rica" EUNED, San José.

Holdridge, L. 1971 "Forest enviroment in tropical life zones. A pilot study". Pergamon Press, Londres.

ICAFE 1992 “Compendio de estadísticas del café” San José.

ICAFE 1993 “Informe sobre la actividad cafetalera de Costa Rica 1993, San José. 
Incer, A. "Contaminación por aguas residuales del beneficio húmedo de café" Ponencia presentada en el seminario "LA cuenca del río Grande de Tárcoles con miras hacia el futuro. San José.

Ramírez, A. Y Maldonado, T. 1988. "Desarrollo socioeconómico y el ambiente natural de Costa Rica” Fundación Neotrópica, San José.

Reynolds, J. 1991. "Soil Nitrogen dynamics in relation to grounwater contamination in the Central Valley, Costa Rica.” Tesis doctoral inédita. Universidad de Michigan.

Rojas, C. 1984. "Estudio de Residuos, Degradación y Comportamiento del Paraquat en Tres Suelos Cafetaleros de Costa Rica." Tesis de Licenciatura en Agronomía, Universidad de Costa Rica.

Rojas, E. 1993. "Efectos de la actual crisis cafetalera en la economía de Costa Rica". Ponencia presentada en el simposio Internacional "Modernización tecnológica, Cambio Social y crisis cafetalera". UNA-ICAFE, Heredia.

Salas, W. 1987. "Efectos de las aguas contaminadas en la producción Agrícola”. Editorial Universidad de Costa Rica, San José.

Sancho, F. 1991. "Medición de pérdidas de suelo a través del empleo de parcelas de escurrimiento". En: "Memoria del taller de erosión de suelos". Escuela de ciencias geográficas, Universidad Nacional Autónoma, Heredia.

IICN 1992. "Evaluación del impacto socio-ambiental de la expansión bananera en Sarapiquí, Tortuguero y Talamanca"

Vargas, J. 1985 "Repercusiones del proceso de metropolización sobre la más antigua zona cafetalera del Valle Central” En Revista de Historia, número especial dedicado al tema "Historia, problemas y perspectiva agraria en Costa Rica. EUNA. Heredia. 


\section{NOTAS}

${ }^{1}$ Al respecto pueden consultarse en el Archivo nacional de Costa Rica, los documentos de la Serie Municipal, números 874 (folio3), 179 (folio 21), 410 (folio 9), 351 (folio 9) y, 22 (folio 31).

${ }^{2}$ El Paraquat es una sustancia extremadamente tóxica, sobre todo por la vía oral. Usado indebidamente, provoca quemaduras en la piel y vías respiratorias, y puede ocasionar intoxicación mortal por contacto con la piel. Causa daños en las uñas, riñones, pulmones, hígado y cerebro. (UICN: 1992: 84)

3 Aunque desde hace tiempo se suponía que la caficultura se había constituido en un importante vector de contaminación de aguas subterráneas en el Valle Central, poco se conocía del grado de efectivo contaminación provocado por la caficultura. Afortunadamente una investigación reciente ha permitido esclarecer el asunto (Reynolds: 1991). A menos que se indique lo contrario el resto de la sección tiene como base dicha investigación. 